\title{
BILIPSCHITZ MAPPINGS WITH DERIVATIVES OF BOUNDED VARIATION
}

\author{
StANislaV HenCl
}

Abstract

Let $\Omega \subset \mathbb{R}^{n}$ be open and suppose that $f: \Omega \rightarrow \mathbb{R}^{n}$ is a bilipschitz mapping such that $D f \in B V_{\text {loc }}\left(\Omega, \mathbb{R}^{n^{2}}\right)$. We show that under these assumptions the inverse satisfies $D f^{-1} \in B V_{\text {loc }}\left(f(\Omega), \mathbb{R}^{n^{2}}\right)$.

\section{Introduction}

Suppose that $\Omega \subset \mathbb{R}^{n}$ is an open set and let $f: \Omega \rightarrow f(\Omega) \subset \mathbb{R}^{n}$ be a homeomorphism. In this paper we address the issue of the regularity of $f^{-1}$ under regularity assumptions on $f$. The starting point for us is the following very recent result from [4] (see Preliminaries for the definition of the space $B V$ ).

Theorem 1.1. Let $\Omega, \Omega^{\prime} \subset \mathbb{R}^{2}$ be open and suppose that $f: \Omega \rightarrow \Omega^{\prime}$ is a homeomorphism. Then $f \in B V_{\mathrm{loc}}\left(\Omega ; \mathbb{R}^{2}\right)$ if and only if $f^{-1} \in B V_{\mathrm{loc}}\left(\Omega^{\prime} ; \mathbb{R}^{2}\right)$. Moreover, both $f$ and $f^{-1}$ are differentiable almost everywhere.

In the same paper we also studied the conditions that guarantee that $f^{-1} \in B V_{\text {loc }}\left(\Omega, \mathbb{R}^{n}\right)$ in higher dimensions. With some additional assumptions (namely that $f$ is a mapping of finite distortion) it is moreover possible to prove that $f^{-1} \in W_{\text {loc }}^{1,1}\left(\Omega, \mathbb{R}^{n}\right)$ (see [2], [3] and [5]).

In this paper we want to address the issue of regularity of the second derivative of $f^{-1}$. The classical inverse function theorem states that if $f$ is $C^{2}$ and $J_{f}\left(x_{0}\right) \neq 0$ then there is a small neighborhood of $x_{0}$ where $f$ is homeomorphism and $f^{-1}$ is $C^{2}$. We will assume that $f$ is a bilipschitz mapping and show that $D f^{-1} \in B V_{\text {loc }}$ provided that $D f \in$ $B V_{\text {loc }}$. This resembles the result from $[6]$ that the inverse of bilipschitz Delta-convex mapping is again Delta-convex.

2000 Mathematics Subject Classification. 26 B30.

Key words. Functions of bounded variation, inverse.

The author was supported in part by GAČR 201/06/P100 and in part by MSM 0021620839 . 
Theorem 1.2. Let $\Omega, \Omega^{\prime} \subset \mathbb{R}^{n}$ be open and suppose that $f: \Omega \rightarrow \Omega^{\prime}$ is a bilipschitz mapping such that $D f \in B V_{\mathrm{loc}}\left(\Omega ; \mathbb{R}^{n^{2}}\right)$. Then $D f^{-1} \in$ $B V_{\text {loc }}\left(\Omega^{\prime} ; \mathbb{R}^{n^{2}}\right)$.

It is moreover possible to show that $D f^{-1}$ belongs to the Sobolev space $W_{\mathrm{loc}}^{1, p}$ if $D f \in W_{\mathrm{loc}}^{1, p}$.

Theorem 1.3. Let $\Omega, \Omega^{\prime} \subset \mathbb{R}^{n}$ be open, $p \geq 1$ and suppose that $f: \Omega \rightarrow$ $\Omega^{\prime}$ is a bilipschitz mapping such that $D f \in W_{\mathrm{loc}}^{1, p}\left(\Omega ; \mathbb{R}^{n^{2}}\right)$. Then $D f^{-1} \in$ $W_{\text {loc }}^{1, p}\left(\Omega^{\prime} ; \mathbb{R}^{n^{2}}\right)$.

Let us make a comment on our assumptions. Let $\alpha>1$ and consider the function $f:(-1,1) \rightarrow \mathbb{R}$ defined as $f(x)=|x|^{\alpha} \operatorname{sgn} x$. Then it is easy to check that $f$ is Lipschitz, homeomorphism, $D f \in W^{1,1}((-1,1))$, but $D f^{-1} \notin B V_{\text {loc }}((-1,1))$. Thus the assumption that $f^{-1}$ is Lipschitz cannot be omitted.

In Section 4 we give an example which shows that Theorem 1.2 is not valid in dimension $n \geq 4$ without the assumption that $f$ is Lipschitz. If $n=1$, then $D f \in B V$ implies that $D f$ is bounded and that $f$ is Lipschitz, and thus this assumption is redundant. We would like to know if a homeomorphism $f: \Omega \rightarrow \Omega^{\prime}$ such that $f^{-1}$ is Lipschitz and $D f \in B V_{\text {loc }}\left(\Omega ; \mathbb{R}^{n^{2}}\right)$ must satisfy $D f^{-1} \in B V_{\text {loc }}\left(\Omega^{\prime} ; \mathbb{R}^{n^{2}}\right)$ in dimensions $n=2$ and $n=3$. Unfortunately our method of the proof and our counterexample do not provide an answer to this question.

\section{Preliminaries}

By $\mathbf{e}_{1}, \ldots, \mathbf{e}_{n}$ we denote the canonical basis in $\mathbb{R}^{n}$. For $x \in \mathbb{R}^{n}$ we write $x_{1}, \ldots, x_{n}$ for its coordinates, i.e. $x=\sum_{i=1}^{n} x_{i} \mathbf{e}_{i}$. The euclidean distance of $x, y \in \mathbb{R}^{n}$ is denoted by $|x-y|$ and the norm of the $n$ times $n$ matrix $A$ is denoted by $\|A\|$.

In the whole paper $\Omega$ will denote an open subset of $\mathbb{R}^{n}$. We say that $F: \Omega \rightarrow \mathbb{R}^{n}$ is a Lipschitz map if there is a constant $K>0$ such that

$$
|F(x)-F(y)| \leq K|x-y|
$$

for every $x, y \in \Omega$. Further $F$ is said to be bilipschitz if it is an invertible mapping and both $F: \Omega \rightarrow \mathbb{R}^{n}$ and $F^{-1}: F(\Omega) \rightarrow \mathbb{R}^{n}$ are Lipschitz.

The Lebesgue measure of a set $A \subset \mathbb{R}^{n}$ is denoted by $\mathcal{L}_{n}(A)$. A mapping $f: \Omega \rightarrow \mathbb{R}^{n}$ is said to satisfy the Lusin condition $(N)$ if $\mathcal{L}_{n}(f(A))=0$ for every $A \subset \Omega$ such that $\mathcal{L}_{n}(A)=0$.

Let $\Omega \subset \mathbb{R}^{n}$ be open and $m \in \mathbb{N}$. A function $h \in L^{1}(\Omega)$ is of bounded variation, $h \in B V(\Omega)$, if the distributional partial derivatives of $h$ are 
measures with finite total variation in $\Omega$ : there are Radon (signed) measures $\mu_{1}, \ldots, \mu_{n}$ defined in $\Omega$ so that for $i=1, \ldots, n,\left|\mu_{i}\right|(\Omega)<\infty$ and

$$
\int_{\Omega} h D_{i} \varphi d x=-\int_{\Omega} \varphi d \mu_{i}
$$

for all $\varphi \in C_{0}^{\infty}(\Omega)$. We say that $f \in L^{1}\left(\Omega, \mathbb{R}^{m}\right)$ belongs to $B V\left(\Omega, \mathbb{R}^{m}\right)$ if the coordinate functions of $f$ belong to $B V(\Omega)$. Analogously we define the Sobolev space: $f \in W^{1,1}\left(\Omega, \mathbb{R}^{m}\right)$ if $f \in L^{1}\left(\Omega, \mathbb{R}^{m}\right)$ and the distributional derivatives of the coordinate functions are in $L^{1}\left(\Omega, \mathbb{R}^{n}\right)$. Further, $f \in B V_{\text {loc }}\left(\Omega, \mathbb{R}^{m}\right)\left(\right.$ or $\left.f \in W_{\text {loc }}^{1,1}\left(\Omega, \mathbb{R}^{m}\right)\right)$ requires that $f \in B V\left(\Omega^{\prime}, \mathbb{R}^{m}\right)$ (or $f \in W^{1,1}\left(\Omega^{\prime}, \mathbb{R}^{m}\right)$ ) for each open $\Omega^{\prime} \Subset \Omega$. For an introduction to the theory of $B V$ and $W^{1,1}$ spaces see [1], [7]. The function $h: \Omega \rightarrow \mathbb{R}^{m}$ is said to be a representative of $g: \Omega \rightarrow \mathbb{R}^{m}$ if $h=g$ almost everywhere with respect to Lebesgue measure.

For function $f:(a, b) \rightarrow \mathbb{R}^{m}$ we define

$$
\begin{array}{r}
V(f,(a, b)):=\sup \left\{\sum_{i=1}^{k}\left|f\left(a_{i}\right)-f\left(b_{i}\right)\right|:\left(a_{i}, b_{i}\right)\right. \text { are pairwise disjoint } \\
\text { intervals in }(a, b)\} .
\end{array}
$$

The function $f$ is said to have finite variation if $V(f,(a, b))<\infty$.

It is a well-known fact (see e.g. [1, Section 3.11]) that a mapping $u \in$ $L_{\text {loc }}^{1}\left(\Omega, \mathbb{R}^{m}\right)$ is in $B V_{\text {loc }}\left(\Omega, \mathbb{R}^{m}\right)$ (or in $W_{\text {loc }}^{1,1}\left(\Omega, \mathbb{R}^{m}\right)$ ) if and only if there is a representative which has bounded variation (or is an absolutely continuous function) on almost all lines parallel to coordinate axes and the variation on these lines is integrable. More precisely, let $i \in\{1,2, \ldots, n\}$ and denote by $\pi_{i}$ the projection on the hyperplane perpendicular to the $x_{i}$-axis. Suppose that $Q(c, r):=\left(c_{1}-r, c_{1}+r\right) \times \cdots \times\left(c_{n}-r, c_{n}+r\right) \subset \Omega$ for some $c \in \mathbb{R}^{n}, r>0$ and set $Q^{i}(c, r)=\pi_{i}(Q(c, r))$. Let $y \in Q^{i}(c, r)$ and denote

$$
u_{i, y}(t)=u\left(y+t \mathbf{e}_{i}\right) \text { for } t \in\left(c_{i}-r, c_{i}+r\right) .
$$

Theorem 2.1. Let $\Omega \subset \mathbb{R}^{n}$ be open and let $u \in L_{\mathrm{loc}}^{1}\left(\Omega, \mathbb{R}^{m}\right)$.

(i) Then $u \in W_{\text {loc }}^{1,1}\left(\Omega, \mathbb{R}^{m}\right)$ if and only if the following happens. For every cube $Q(c, r) \Subset \Omega$ and for every $i \in\{1, \ldots, n\}$ there is a representative $\tilde{u}$ of $u$ such that the function $\tilde{u}_{i, y}(t)$ is absolutely continuous on $\left(c_{i}-r, c_{i}+r\right)$ (i.e. each coordinate function is absolutely 
continuous) for $\mathcal{L}_{n-1}$ almost every $y \in Q^{i}(c, r)$ and moreover

$$
\int_{Q^{i}(c, r)} \int_{c_{i}-r}^{c_{i}+r}\left|\nabla \tilde{u}_{i, y}(t)\right| d t d y<\infty .
$$

(ii) Then $u \in B V_{\text {loc }}\left(\Omega, \mathbb{R}^{m}\right)$ if and only if the following happens. For every cube $Q(c, r) \Subset \Omega$ and for every $i \in\{1, \ldots, n\}$ there is a representative $\tilde{u}$ of $u$ such that the function $\tilde{u}_{i, y}(t)$ has bounded variation on $\left(c_{i}-r, c_{i}+r\right)$ for $\mathcal{L}_{n-1}$ almost every $y \in Q^{i}(c, r)$ and moreover

$$
\int_{Q^{i}(c, r)} V\left(\tilde{u}_{i, y},\left(c_{i}-r, c_{i}+r\right)\right) d y<\infty .
$$

We shall also need that the composition of $B V$ function and a homeomorphism with Lipschitz inverse is in $B V$ (see [1, Theorem 3.16 and Corollary 3.19]).

Theorem 2.2. Let $\Omega, \Omega^{\prime} \subset \mathbb{R}^{n}$ be open and let $u: \Omega \rightarrow \mathbb{R}^{m}$. Suppose that $F: \Omega \rightarrow \Omega^{\prime}$ is Lipschitz and homeomorphism.

(i) If $u \in B V_{\text {loc }}\left(\Omega, \mathbb{R}^{m}\right)$, then $u \circ F^{-1} \in B V\left(\Omega^{\prime}, \mathbb{R}^{m}\right)$.

(ii) If $u \in W_{\mathrm{loc}}^{1,1}\left(\Omega, \mathbb{R}^{m}\right)$ and $F^{-1}$ is Lipschitz, then $u \circ F^{-1} \in W_{\mathrm{loc}}^{1,1}\left(\Omega^{\prime}, \mathbb{R}^{m}\right)$ and

$D u \circ F^{-1}(y)=D u\left(F^{-1}(y)\right) D F^{-1}(y)$ for almost every $y \in \Omega^{\prime}$.

\section{Regularity of the inverse}

Proof of Theorem 1.2: We want to show that $D f^{-1}$ has bounded variation on almost all lines parallel to coordinate axes and therefore $D f^{-1} \in$ $B V_{\text {loc }}$ (see Theorem 2.1 (ii)). Fix $Q(c, r) \Subset f(\Omega)$ and $i \in\{1, \ldots, n\}$.

From Theorem 2.2 we know that $D f \circ f^{-1} \in B V_{\text {loc }}$. Denote by $h$ a good representative of $D f \circ f^{-1}$ from Theorem 2.1 (ii) and set $h_{i, y}(t):=$ $h\left(y+t \mathbf{e}_{i}\right)$ for $y \in Q^{i}(c, r)$. From (2.2) we have

$$
\int_{Q^{i}(c, r)} V\left(h_{i, y},\left(c_{i}-r, c_{i}+r\right)\right) d y<\infty .
$$

Denote

$$
\begin{array}{r}
A=\left\{x \in Q(c, r): h(x)=D f \circ f^{-1}(x), f^{-1} \text { is differentiable at } x\right. \\
\text { and } \left.f \text { is differentiable at } f^{-1}(x)\right\} .
\end{array}
$$

Lipschitz functions are differentiable almost everywhere and map Lebesgue null sets to Lebesgue null sets and therefore $\mathcal{L}_{n}(A)=\mathcal{L}_{n}(Q(c, r))$. From the definition of $A$ we have

$$
D f^{-1}(x) D f\left(f^{-1}(x)\right)=I \text { for every } x \in A .
$$


Fix $y \in Q^{i}(c, r)$ and let $\left\{\left(a_{j}, b_{j}\right)\right\}_{j=1}^{k}$ be a system of pairwise disjoint subintervals of $\left(c_{i}-r, c_{i}+r\right)$ such that $A_{j}:=y+a_{j} \mathbf{e}_{i} \in A$ and $B_{j}:=$ $y+b_{j} \mathbf{e}_{i} \in A$ for every $j$. Plainly $\left\|D f^{-1}(x)\right\| \leq K$ where $K$ denotes the Lipschitz constant of $f^{-1}$. Together with (3.2) this imply

$$
\begin{aligned}
\sum_{j=1}^{k} & \left\|D f^{-1}\left(A_{j}\right)-D f^{-1}\left(B_{j}\right)\right\| \\
& =\sum_{j=1}^{k}\left\|D f^{-1}\left(A_{j}\right)\left(D f\left(f^{-1}\left(B_{j}\right)\right)-D f\left(f^{-1}\left(A_{j}\right)\right)\right) D f^{-1}\left(B_{j}\right)\right\| \\
& \leq K^{2} \sum_{j=1}^{k}\left\|D f\left(f^{-1}\left(B_{j}\right)\right)-D f\left(f^{-1}\left(A_{j}\right)\right)\right\| \\
& \leq C V\left(h_{i, y},\left(c_{i}-r, c_{i}+r\right)\right) .
\end{aligned}
$$

From (3.1) and $\mathcal{L}_{n}(A)=\mathcal{L}_{n}(Q(c, r))$ we know that $V\left(h_{i, y},\left(c_{i}-r, c_{i}+\right.\right.$ $r))<\infty$ and $\mathcal{L}_{1}\left(\pi_{i}^{-1}(y) \cap A\right)=2 r$ for $\mathcal{L}_{n-1}$ almost every $y$. Fix such a $y \in Q^{i}(c, r)$. From (3.3) and elementary properties of functions of bounded variation we obtain that there is a function $\tilde{u}_{i, y}:\left(c_{i}-r, c_{i}+r\right) \rightarrow$ $\mathbb{R}^{n^{2}}$ such that $D f^{-1}\left(y+t \mathbf{e}_{i}\right)=\tilde{u}_{i, y}(t)$ for every $t \in\left(c_{i}-r, c_{i}+r\right) \cap A$ and

$$
V\left(\tilde{u}_{i, y},\left(c_{i}-r, c_{i}+r\right)\right) \leq C V\left(h_{i, y},\left(c_{i}-r, c_{i}+r\right)\right) .
$$

It follows that there is a function $\tilde{u}$ such that $\tilde{u}(x)=D f^{-1}(x)$ almost everywhere and this new representative has bounded variation on $Q(c, r) \cap \pi_{i}^{-1}(y)$ for $\mathcal{L}_{n-1}$ almost every $y \in Q^{i}(c, r)$. Now (3.4) and (3.1) yields

$$
\int_{Q^{i}(c, r)} V\left(\tilde{u}_{i, y},\left(c_{i}-r, c_{i}+r\right)\right) d y<\infty
$$

which verifies (2.2) for $D f^{-1}$.

Proof of Theorem 1.3: First let us prove the theorem in the case $p=1$. The proof of this case is analogous to the previous proof and therefore we only sketch it and point out the differences. From Theorem 2.2 (ii) we know that $D f \circ f^{-1} \in W_{\text {loc }}^{1,1}$. Fix $y \in Q^{i}(c, r)$ such that $h_{i, y}$ is absolutely continuous on $\left(c_{i}-r, c_{i}+r\right)$. Given $\varepsilon>0$ find $\delta>0$ from the absolute continuity of $h_{i, y}$. Choose $A_{j}$ and $B_{j}$ as before and moreover assume 
that $\sum_{j=1}^{k}\left|A_{j}-B_{j}\right|<\delta$. Analogously to (3.3) we obtain

$$
\sum_{j=1}^{k}\left\|D f^{-1}\left(A_{j}\right)-D f^{-1}\left(B_{j}\right)\right\|<C \varepsilon
$$

Reasoning analogously to the previous proof we conclude that $D f^{-1}$ has a representative which is absolutely continuous on almost all lines parallel to coordinate axes. On those lines we have

$$
V\left(\tilde{u}_{i, y},\left(c_{i}-r, c_{i}+r\right)\right)=\int_{c_{i}-r}^{c_{i}+r}\left|\nabla \tilde{u}_{i, y}(t)\right| d t .
$$

From Theorem 1.2 and Theorem 2.1 (ii) we already know (2.2) and thus we obtain (2.1).

Now let us return to the case $p>1$. We already know that $D f^{-1} \in$ $W_{\text {loc }}^{1,1}$. Therefore we can use Theorem 2.2 (ii) and differentiate twice the identity $f \circ f^{-1}(y)=y$ to obtain

$$
D^{2} f\left(f^{-1}(y)\right) D f^{-1}(y) D f^{-1}(y)+D f\left(f^{-1}(y)\right) D^{2} f^{-1}(y)=0 .
$$

Here and in the sequel we identify the second derivative with an linear operator from $\mathbb{R}^{n^{2}}$ to $\mathbb{R}^{n^{2}}$. Clearly

$$
\left\|\left(D f\left(f^{-1}(y)\right)\right)^{-1}\right\| \leq C,\left\|D f^{-1}(y)\right\| \leq C \text { and }\left|J_{f^{-1}}(y)\right| \geq C
$$

at almost every point since $f$ is bilipschitz. From (3.6) and substitution formula we now obtain

$$
\begin{aligned}
\int_{A}\left\|D^{2} f^{-1}(y)\right\|^{p} d y & \leq C \int_{A}\left\|D^{2} f\left(f^{-1}(y)\right)\right\|^{p}\left|J_{f^{-1}}(y)\right| d y \\
& =C \int_{f^{-1}(A)}\left\|D^{2} f(x)\right\|^{p} d x
\end{aligned}
$$

for every open set $A \Subset f(\Omega)$ and the claim follows.

\section{Necessity of the Lipschitz condition for $f$ for $n \geq 4$}

Example 4.1. Let $n \geq 4$. There is a homeomorphism $f:(-1,1)^{n} \rightarrow \mathbb{R}^{n}$ such that $D f \in W^{1,1}\left((-1,1)^{n}, \mathbb{R}^{n^{2}}\right)$ and $f^{-1}$ is Lipschitz, but $D f^{-1} \notin$ $B V_{\text {loc }}\left(f\left((-1,1)^{n}\right), \mathbb{R}^{n^{2}}\right)$.

Proof: Given $x \in \mathbb{R}^{n}$ we denote $\tilde{x}=\left[x_{1}, \ldots, x_{n-1}\right] \in \mathbb{R}^{n-1}$ and $\|\tilde{x}\|=$ $\sqrt{x_{1}^{2}+\cdots+x_{n-1}^{2}}$. 
Let $\alpha=\frac{1}{2 n}, \beta=\frac{3}{4}$ and set

$$
f(x)=\sum_{i=1}^{n-1} \mathbf{e}_{i} x_{i}\|\tilde{x}\|^{\alpha-1}+\mathbf{e}_{n}\left(x_{n}+\|\tilde{x}\| \sin \left(\|\tilde{x}\|^{-\beta}\right)\right)
$$

if $\|\tilde{x}\|>0$ and $f(x)=\mathbf{e}_{n} x_{n}$ if $\|\tilde{x}\|=0$. Our mapping $f$ is clearly continuous and it is easy to check that $f$ is a one-to-one map since

$$
\begin{aligned}
& x_{i}\|\tilde{x}\|^{\alpha-1}=z_{i}\|\tilde{z}\|^{\alpha-1} \text { for every } i \in\{1, \ldots, n-1\} \Rightarrow \\
& \Rightarrow x_{i}=z_{i} \text { for every } i \in\{1, \ldots, n-1\} .
\end{aligned}
$$

Therefore $f$ is a homeomorphism.

A direct computation shows that the second partial derivatives of $f_{i}$, $i \in\{1, \ldots, n-1\}$, are smaller than $C\|\tilde{x}\|^{\alpha-2}$ and therefore integrable. Moreover,

$$
\frac{\partial f_{n}(x)}{\partial x_{1}}=x_{1}\|\tilde{x}\|^{-1} \sin \left(\|\tilde{x}\|^{-\beta}\right)-\|\tilde{x}\| \beta \frac{x_{1}}{\|\tilde{x}\|^{\beta+2}} \cos \left(\|\tilde{x}\|^{-\beta}\right) .
$$

It is not difficult to compute that we can bound each partial derivative of this expression by $C\|\tilde{x}\|^{1-2(\beta+1)}$. Clearly $2(\beta+1)-1<n-1$ and therefore these second partial derivatives are integrable. Analogously we can estimate other second partial derivatives of $f_{n}$. Since the second derivatives of $f$ are smooth outside the segment $\{[0, \ldots, 0, t]: t \in(-1,1)\}$ and $\left|D^{2} f\right| \in L^{1}\left((-1,1)^{n}\right)$ it is easy to see that $D f \in W^{1,1}\left((-1,1)^{n}, \mathbb{R}^{n^{2}}\right)$.

The inverse of $f$ is given by

$$
f^{-1}(y)=\sum_{i=1}^{n-1} \mathbf{e}_{i} y_{i}\|\tilde{y}\|^{\frac{1}{\alpha}-1}+\mathbf{e}_{n}\left(y_{n}-\|\tilde{y}\|^{\frac{1}{\alpha}} \sin \left(\|\tilde{y}\|^{-\frac{\beta}{\alpha}}\right)\right)
$$

if $\|\tilde{y}\|>0$ and $f^{-1}(y)=\mathbf{e}_{n} y_{n}$ if $\|\tilde{y}\|=0$. The derivative of the function $\phi(t)=t^{\frac{1}{\alpha}} \sin \left(t^{-\frac{\beta}{\alpha}}\right)$ is bounded on $(-1,1)$ and therefore $\phi$ is Lipschitz. Thus it is not difficult to see that $f^{-1}$ is Lipschitz.

The second derivative of $f^{-1}$ is clearly continuous outside the segment $\{[0, \ldots, 0, t]: t \in \mathbb{R}\}$. Elementary computation gives us

$$
\frac{\partial\left(f^{-1}\right)_{n}(y)}{\partial y_{1}}=-\frac{y_{1}}{\alpha}\|\tilde{y}\|^{\frac{1}{\alpha}-2} \sin \left(\|\tilde{y}\|^{-\frac{\beta}{\alpha}}\right)+\|\tilde{y}\|^{\frac{1}{\alpha}} \frac{\beta}{\alpha} \frac{y_{1}}{\|\tilde{y}\|^{\frac{\beta}{\alpha}+2}} \cos \left(\|\tilde{y}\|^{-\frac{\beta}{\alpha}}\right)
$$

and therefore the second derivative $\frac{\partial^{2}\left(f^{-1}\right)_{n}(y)}{\partial y_{1}^{2}}$ contains some integrable terms and

$$
-\|\tilde{y}\|^{\frac{1}{\alpha}} \frac{\beta^{2}}{\alpha^{2}} \frac{y_{1}^{2}}{\|\tilde{y}\|^{2 \frac{\beta}{\alpha}+4}} \sin \left(\|\tilde{y}\|^{-\frac{\beta}{\alpha}}\right) .
$$


Since

$$
2\left(\frac{\beta}{\alpha}+1\right)-\frac{1}{\alpha}>n-1
$$

we obtain that the integral of the absolute value of (4.1) over the set

$$
S=\left\{y \in f\left((-1,1)^{n}\right): y_{1}>\frac{1}{2}\|\tilde{y}\|, \sin \left(\|\tilde{y}\|^{-\frac{\beta}{\alpha}}\right)>\frac{1}{2}\right\}
$$

is infinite. Hence $\left|D^{2} f^{-1}\right| \notin L_{\text {loc }}^{1}$ and it is not difficult to deduce that $D f^{-1} \notin B V_{\mathrm{loc}}$.

Acknowledgements. The author would like to thank to Professor L. Zajíček for suggesting the problem. The author wishes to express his thanks to Professor J. Malý for many stimulating conversations and for helpful suggestions.

\section{References}

[1] L. Ambrosio, N. Fusco, And D. Pallara, "Functions of bounded variation and free discontinuity problems", Oxford Mathematical Monographs, The Clarendon Press, Oxford University Press, New York, 2000.

[2] S. Hencl and P. Koskela, Regularity of the inverse of a planar Sobolev homeomorphism, Arch. Ration. Mech. Anal. 180(1) (2006), $75-95$.

[3] S. Hencl, P. Koskela, and J. Malý, Regularity of the inverse of a Sobolev homeomorphism in space, Proc. Roy. Soc. Edinburgh Sect. A 136(6) (2006), 1267-1285.

[4] S. Hencl, P. Koskela, and J. Onninen, Homeomorphisms of bounded variation, Arch. Ration. Mech. Anal. (to appear).

[5] J. Onninen, Regularity of the inverse of spatial mappings with finite distortion, Calc. Var. Partial Differential Equations 26(3) (2006), $331-341$.

[6] L. VeselÝ And L. ZaJíčEK, Delta-convex mappings between Banach spaces and applications, Dissertationes Math. (Rozprawy Mat.) 289 (1989), $52 \mathrm{pp}$.

[7] W. P. Ziemer, "Weakly differentiable functions. Sobolev spaces and functions of bounded variation", Graduate Texts in Mathematics 120, Springer-Verlag, New York, 1989. 
Department of Mathematical Analysis Charles University

Sokolovská 83

18600 Prague 8

Czech Republic

E-mail address: hencl@karlin.mff.cuni.cz

Primera versió rebuda el 26 de setembre de 2006 , darrera versió rebuda el 21 de març de 2007. 